\title{
A Study on Evaluation of Different Culture Media for the Isolation of Routine Urinary Pathogens
}

\author{
Biji, S. Manjusree*, O. Sasikumari and J.T. Ramani Bai \\ Department of Microbiology Government Medical College, Thiruvananthapuram, India \\ *Corresponding author
}

\section{A B S T R A C T}

\begin{tabular}{|l|}
\hline Ke y w o r d s \\
Urinary Pathogens, \\
Different Culture \\
Media \\
\hline Article Info \\
\hline $\begin{array}{l}\text { Accepted: } \\
\text { 12 January } 2017 \\
\text { Available Online: } \\
\text { 10 February } 2017\end{array}$ \\
\hline
\end{tabular}

Urinary Tract Infections are among the most common bacterial infections and account for a significant part of the workload in clinical microbiology laboratories. The rate of yield is almost same for all media i.e., Blood Agar, MacConkey gar, C.L.E.D Agar with Andrade's indicator and Hichrome UTI Agar. The results obtained after various specific biochemical tests and other techniques, were correlated correctly with the presumptive identification results by Hicrome UTI Agar. Presumptive identification of isolates is a task that requires a great deal of experience when using traditional media like blood agar and Mac Conkey agar. On chromogenic media, this is easier, thus requiring less training. Thus, the use of chromogenic media may improve the quality of urine culture by contributing to a more uniform interpretation of urine culture plates by the different personnel engaged in this task at the laboratory. Blood agar should continue to be a part of the urine culture workup, as isolation and discrimination of different Gram-positive bacteria species are much easier with this medium. As many of the extra tests for bacterial identification associated with conventional culture methods were no longer required, chromogenic medium substantially reduced the laboratory workload. For detection of mixed bacterial growth Hichrome UTI Agar precedes the other media. The excessive use of Blood agar can be minimised in $24 \mathrm{hr}$ working microbiology laboratories. Therefore Hichrome UTI Agar or C.L.E.D Agar with Andrade's indicator is advisable as primary plating media. HiChrome UTI agar was found to be more useful as primary urine culture medium in both higher rate of isolation and presumptive identification of uropathogens in comparison to conventional media. Its inherent characteristics in demonstrating polymicrobial growth and ease of rapid identification by distinct colony colour are unique. Improved detection of mixed cultures may help to identify contaminated specimens and therefore lead to a reduction in the prescription of unnecessary antibiotics. The overall findings of this study suggests and reinforced that HiCrome UTI agar offers an excellent and time saving method for the reliable identification of most of the uropathogens and differentiation of mixed bacterial cultures in primary culture plate as opposed to conventional culture system. This study suggests and reinforced that HiCrome UTI agar offers an excellent and time saving method for the reliable identification of most of the uropathogens and differentiation of mixed bacterial cultures in primary culture plate as opposed to conventional culture system. It has the potential to streamline urine culture processing in a meaningful way, such as reducing technologist workload, improving result turnaround times which together all have considerable laboratory impact. 


\section{Introduction}

Urinary tract infection (UTI) is one of the most common conditions causing individuals to seek medical care. Urinary tract infections are important because they cause acute morbidity and may reduce renal function. In almost all cases there is a need to start treatment before the final microbiological results are obtained.

The laboratory diagnosis of urinary tract infection requires semi quantitative urine culture on standard agar media. As only 20 to $30 \%$ of urine samples result in significant growth, a considerable amount of time is spent evaluating samples that do not have clinical utility. The increase in resistance of microorganisms to antimicrobial agents, especially in hospitalized patients, demands rapid identification of the pathogen. Early information enables the selection of the appropriate antibiotics prior to the results of susceptibility tests and may thereby prevent outbreaks. Therefore any new medium or method with the ability to streamline urine culture processing in a meaningful way would be clinically useful and has the potential to have considerable laboratory Impact (Kanchana et al., 2013).

For many years Blood Agar and MacConkey Agar have been used for the detection of urinary tract pathogens. Careful observers are often able to recognize mixtures of different gram negative bacteria when they occur on a single plate. A recently developed medium, HiChrome UTI agar, incorporates chromogenic substrates for the detection of colonies from mixed cultures and single step identification of organisms. Thus it decreases the work $\operatorname{load}^{2}$. In the present study the efficacy of HiChrome UTI agar was evaluated in the central microbiology laboratory as a primary urine culture medium for its rate of isolation and presumptive identification of uropathogens in comparison with a nonchromogenic medium, blood agar, Mac Conkey agar and Cystein Lactose Electrolyte Deficient Agar in terms of isolation rate and to detect mixed cultures for the diagnosis of urinary tract infection.

\section{Materials and Methods}

Design of study: Descriptive study

Study setting: Central Microbiology Laboratory, Medical College Hospital, Thiruvananthapuram, Department of MLT, Govt. Medical college, Thiruvananthapuram.

Study population: Urine samples received in central microbiology lab from out patients and inpatients

Inclusion criteria: All urine samples in sterile urine culture bottle.

Exclusion criteria: Urine samples in unsterile containers.

Sample size: 353

Duration of study: 6 months

Study setting: Clinical Microbiology Laboratory at Govt. Medical College Hospital, Thiruvananthapuram, Kerala, in India.

\section{Procedure}

353 urine samples were tested. Samples were collected in sterile containers. The urine samples were inoculated on Blood Agar, MacConkey Agar, Cysteine Lactose Electrolyte Deficient Agar (CLED) with Andrade's indicator and Hicrome UTI Agar. The inoculated plates were incubated at $37^{\circ} \mathrm{c}$ overnight. Presumptive identification of bacterial growth was done on HiCrome UTI agar according to colony morphology and 
color as depicted by the manufacturer. Colonies on the MacConkey agar, CLED Agar and BA were also identified following colony characteristics against each of the uropathogens. The final identification of the isolates was done using standard identification protocol such as Gram's staining, motility test, catalase test, coagulase test, oxidase test and other relevant biochemical tests as appropriate for the isolates.

\section{Results and Discussion}

The present study evaluated the ability of Blood Agar, MacConkey Agar, Hicrome UTI Agar and CLED Agar with Andrade's indicator for detection and identification of routine urinary pathogens. Out of 353 urine samples, $123(34.84 \%)$ yielded significant bacterial growth, $20(5.66 \%)$ showed no significant bacterial growth, 60 (16.99\%) showed mixed bacterial growth, and 150 $(42.49 \%)$ showed no growth. Only 35\% showed significant bacterial growth. According to the studies by Salvatore et al., (2011), Parveen et al., and Sharmin et al., (2010), only 40-50\% showed significant bacterial growth (Salvatore et al., 2011; Parveen et al., 2011; Sharmin et al., 2010). A higher rate of isolation was reported from a study in UK (54.2\% single growth and 21.6\% mixed growth) by Perry and Freydiere, (2007).

The isolates from urine culture enlisted in table 2. The major isolate was Escherichia coli $(37 \%)$, followed by Klebsiella species $(21 \%)$. Other isolates were Candida species (12\%), Enterococcus spp. (11\%), Pseudomonas aeruginosa (10\%), Staphylococcus aureus (4\%), Acinetobacter (4\%) and Serratia species (1\%). According to the studies by Salvatore et al., (2011), Parveen et al., (2011) 51 and Sharmin et al., (2010) predominant growth was Escherichia coli (Salvatore et al., 2011; Parveen et al.,
2011; Sharmin et al., 2010). Due to the specific color and colony morphology on Hicrome UTI Agar the identification of each organism is easier. Escherichia coli produced pink colored colonies on Hicrome UTI Agar. Klebsiella spp and Serratia produced metallic blue colored colonies on chromogenic agar. Pseudomonas aeruginosa produced creamy translucent colonies (greenish pigmented). Acinetobacter spp produced round cream colored colonies. Staphylococcus aureus produced glistening cream colored colonies. Enterococci produced turquoise blue colored colonies. Candida spp produced white or cream colored colonies. The results showed that overnight incubation is optimal for the growth response of microorganisms on HiChrome UTI agar medium. Longer incubation of up to $72 \mathrm{hrs}$ confirmed the results and deepened the colony colors.

Table 3 shows the rate of isolation of mixed growth. Out of 60 mixed bacterial growth all (100\%) were detected by Hicrome UTI Agar on second day itself; 50\%by Blood Agar, $58 \%$ by MacConkey's Agar and $60 \%$ by CLED Agar with Andrade's indicator. Detection of mixed bacterial growth is easier in Hicrome UTI Agar. Previous studies by Lakshmi et al., (2004) and Perry and Freydiere (2007) suggested that best advantage of chromogenic media over other media was easy recognition of mixed growth and preventing the need for subculturing and performing multiple biochemical tests (Perry et al., 2007). Cystein Lactose Electrolyte Deficient Agar with Andrade's indicator showed colour changes depending on the production of acid by different organisms.

Table 4 shows the ability of HiChrome UTI agar medium (100\%) to detect uropathogens. It is equal to that of the combination of the two reference media blood agar and MacConkey Agar. CLED Agar with Andrade's indicator also showed good response in detection of uropathogens. The 
rate of isolation of uropathogens of the present study are in accordance with few studies by Parveen et al., (2011), Sharmin (2010) et al., Ciragil et al., (2006), and Rani et al., in Bangalore, India (2012) carried out on both chromogenic and conventional media.
HiCrome UTI agar, Blood agar and CLED Agar with Andrade's indicator supported the growths of all $123(100 \%)$ isolates whereas MacConkey agar yielded 110(97.56\%) bacterial growths.

Table.1 Growth of Result of urine culture

\begin{tabular}{|l|l|l|}
\hline Type of Growth & Number & $\%$ \\
\hline $\begin{array}{l}\text { Significant bacterial } \\
\text { growth }\end{array}$ & 123 & 35 \\
\hline $\begin{array}{l}\text { No Significant } \\
\text { bacterial growth }\end{array}$ & 20 & 6 \\
\hline Mixed bacterial growth & 60 & 17 \\
\hline No Growth & 150 & 42 \\
\hline Total & 353 & 100 \\
\hline
\end{tabular}

Table.2 Showing number of isolates

\begin{tabular}{|l|l|l|}
\hline Name of the isolate & Number & \% \\
\hline Escherichia coli & 45 & 36.58 \\
\hline Klebsiella spp. & 26 & 21.13 \\
\hline Serratia species & 1 & 0.81 \\
\hline Candida species & 15 & 12.19 \\
\hline $\begin{array}{l}\text { Pseudomonas } \\
\text { aeruginosa }\end{array}$ & 12 & 9.75 \\
\hline Enterococcus spp. & 14 & 11.38 \\
\hline $\begin{array}{l}\text { Staphylococcus } \\
\text { aureus }\end{array}$ & 5 & 4.06 \\
\hline Acinetobacter spp. & 5 & 4.06 \\
\hline Total & 123 & 100 \\
\hline
\end{tabular}


Table.3 Showing rate of detection of mixed bacterial growth in media

\begin{tabular}{|l|l|l|}
\hline Media & Number & \% \\
\hline Blood agar & 30 & 50 \\
\hline Mac conkey agar & 35 & 58 \\
\hline CLED & 36 & 60 \\
\hline Hicrome UTI agar & 60 & 100 \\
\hline
\end{tabular}

Table.4 Comparison of four culture media for the rate of isolation of uropathogens

\begin{tabular}{|l|l|l|l|l|}
\hline Isolate no & Blood agar N \% & Mac conkey N\% & CLED N\% & Hicrome N\% \\
\hline Escherichia coli 45 & $45(100)$ & $45(100)$ & $45(100)$ & $45(100)$ \\
\hline Klebsiella spp.26 & $26(100)$ & $26(100)$ & $26(100)$ & $26(100)$ \\
\hline Serratia species 1 & $1(100)$ & $1(100)$ & $1(100)$ & $1(100)$ \\
\hline Candida species 15 & $15(100)$ & $8(53.3)$ & $15(100)$ & $15(100)$ \\
\hline $\begin{array}{l}\text { Pseudomonas aeruginosa- } \\
\text { 12 }\end{array}$ & $12(100)$ & $12(100)$ & $12(100)$ & $12(100)$ \\
\hline Enterococcus spp.14 & $14(100)$ & $12(85.71)$ & $14(100)$ & $14(100)$ \\
\hline Staphylococcus aureus 5 & $5(100)$ & $4(80)$ & $5(100)$ & $5(100)$ \\
\hline Acinetobacter spp. 5 & $5(100)$ & $5(100)$ & $5(100)$ & $5(100)$ \\
\hline Total & $123(100)$ & $113(91.87)$ & $123(100)$ & $123(100)$ \\
\hline
\end{tabular}

Table.5 Rate of presumptive identification

\begin{tabular}{|l|l|l|l|l|}
\hline Isolate \&total no & $\begin{array}{l}\text { Blood agar } \\
(\boldsymbol{\%})\end{array}$ & $\begin{array}{l}\text { Mac conkey } \\
(\boldsymbol{\%})\end{array}$ & $\begin{array}{l}\text { CLED } \\
(\boldsymbol{\%})\end{array}$ & $\begin{array}{l}\text { Hicrome } \\
(\boldsymbol{\%})\end{array}$ \\
\hline Escherichia coli -45 & $28(62)$ & $30(66)$ & $30(66)$ & $45(100)$ \\
\hline Klebsiella spp-.26 & $11(42)$ & $13(50)$ & $13(50)$ & $26(100)$ \\
\hline Serratia species -1 & $1(100)$ & $1(100)$ & $1(100)$ & $1(100)$ \\
\hline Candida species -15 & $10(66)$ & $7(46)$ & $7(46)$ & $15(100)$ \\
\hline $\begin{array}{l}\text { Pseudomonas } \\
\text { aeruginosa-12 }\end{array}$ & $6(50)$ & $5(41)$ & $5(41)$ & $12(100)$ \\
\hline Enterococcus spp.-14 & $7(50)$ & $7(50)$ & $7(50)$ & $14(100)$ \\
\hline $\begin{array}{l}\text { Staphylococcus } \\
\text { aureus }-5\end{array}$ & $3(60)$ & $2(40)$ & $5(100)$ & $5(100)$ \\
\hline Acinetobacter spp. -5 & 0 & 0 & 0 & $5(100)$ \\
\hline
\end{tabular}


Blood agar is an enriched medium; Cystein Lactose Electrolyte Deficient Agar and HiCrome UTI agar also contains all essential nutrients to support the growth of possible uropathogens that is why all isolates were possible to be grown on to these two media. Similar findings were also reported by Galliot et al., (2000) and Parveen et al., (2011).

The chromogenic media studied can be used as a single primary isolation media for identification of urinary tract pathogens. Several researchers - Aspell et al., (2002), Carricajo et al., (1999), and Scarparo et al., (2002), have previously demonstrated equal or superior performance of various chromogenic media over traditional media for identification of urinary tract pathogens.

Table 5 shows the rate of presumptive identification of isolated organisms. In case of presumptive identification Hicrome UTI Agar precedes the other conventional media. The findings were similar to the study of Kiyofumi Ohkusu (2000).

Other researchers; Samra et al., and engstler et al., speculated that the speed and reliability of identification on chromogenic media may render them cost effective, although the price of chromogenic media is much higher than traditional media (Samra et al., 1998; Hengstler et al., 1997). According to a study by Kiyofumi ohkusu (2000), Japan; an overall reduction of about $70 \%$ in the cost of identification of Gram negative bacilli was achieved. Cost comparison results were based on the commercial cost of each identification kit; but the cost of labour associated with testing was not included.

\section{References}

Aspell, O., Osterman, B., Dttmer, R., Sten, L., Lindback, E., Forsum, U. et al. 2002. Performance of four chromogenic urine culture media after one or two days of incubation compared with reference media. J. Clin. Microbiol., 40: 15001503.

Carricajo, A., Boiste, S., Thore, J., Aubert, G., Gille, Y., Freydiere, A.M. et al. 1999. Comparative evaluation of five chromogenic media for detection, enumeration and identification of urinary tract pathogens. Eur. J. Clin. Microbiol. Infect. Dis., 18: 796-803.

Ciragil, P., Gul, M., Aral, M., Ekerbicer, H. 2006. Evaluation of a new chromogenic medium for isolation and identification of common urinary tract pathogens. Eur. J. Clin. Microbiol. Infect. Dis., 25: 108-1113.

Gaillot, O., Wetsch, M., Fortineau, N., Berche, P. 2000. Evaluation of CHROMagar Staph. aureus, a new chromogenic medium, for isolation and presumptive identification of S. Aureus from human clinical specimens. J. Clin. Microbiol., 38: 1587-1591.

Hengstler, K.A., R. Hammann, and A. Fahr. 1997. Evaluation of BBL CHROMagar Orientation medium for detection and presumptive identification of urinary tract pathogens. J. Clin. Microbiol., 35: 2773-2777.

Kanchana Manickam, James, A., Karlowsky, Heather Adam, Philippe, R.S., LagacéWiens, Assunta Rendina, Paulette Pang, et al. 2013. CHROMagar Orientation Medium Reduces Urine Culture Workload, J. Clin. Microbiol., 51(4): 1179.

Lakshmi, V., Satheeshkumar, T., Kulkarni, G. 2004. Utility of Urichrom II $-\mathrm{A}$ Chromogenic Medium for Uropathogens. Indian J. Med. Microbiol., 22(3): 15.

Leela Rani, Venkata Bharat Kumar Pinnelli, Hemavathi, Sandhya Belwadi, Rajendran, R. 2012. Utility of HiChrome Urinary Tract Infection 
(UTI) Agar Medium for Identification of Uropathogens: A Comparative Study with Other Conventional Media. Int. J. Chem. Pharmaceutical Res., ISSN: 23191716 Volume 1: Issue 4.

Parveen, R., Saha, S.K., Shamshuzzaman, S.M., Rashid, A.L., Chowdhury, A., Muazzam, N. 2011. Detection of Uropathogens by Using Chromogenic Media (Hicrome UTI agar), CLEDagar and other Conventional Media. Faridpur Med. Coll. J., 6(1): 46-50.

Perry, J.D., Freydiere, A.M. 2007. The application of chromogenic media in Clinical Microbiology. J. Appl. Microbiol., 103: 2046-2055.

Rani, L., Pinnelli, V.B.K., Hemavathi, Belwadi, S., Rajendran, R. Utility of HiChrome Urinary Tract Infection (UTI) Agar Medium for Identification of Uropathogens: A ComparativeStudy with Other Conventional Media. $J$. Chem. Pharm. Res.

Salvatore, S., Cattoni, E., Siesto, G., Serati,
M., Sorice, P., Torella, M. et al. 2011. Urinary tract infections in women. Eur. J. Obstet. Gynecol. Reprod. Biol., 156(2): 131-136.

Samra, Z., Heifetz, M., Talmor, J., Bain, z. and Bahar, J. 1998. Evaluation of Use of a New Chromogenic Agar in Detection of Urinary Tract Pathogens. J. Clin. Microbiol., 36(4): 990-994.

Scarparo, C., Piccoli, P., Ricordi, P., Scagnelli, M. Comparative evaluation of two commercial chromogenic media for detection and presumptive identification of urinary tract pathogens. Eur. J. Clin. Microbiol. Infect. Dis., 21: 283-289.

Sharmin, S., Alamgir, F., Begum, F., Jaigirdar, Q. 2010. Use of chromogenic agar media for identification of uropathogen. Bangladesh J. Med. Microbiol., 4: 18-23.

Use of CHROMagar Orientation Medium in Conjunction with Simple Biochemical Tests, J. Clin. Microbiol., 4586-4592.

\section{How to cite this article:}

Biji, S. Manjusree, O. Sasikumari and Ramani Bai, J.T. 2017. A Study on Evaluation of Different Culture Media for the Isolation of Routine Urinary Pathogens. Int.J.Curr.Microbiol.App.Sci. 6(2): 195-201. doi: http://dx.doi.org/10.20546/ijcmas.2017.602.026 